\title{
NUMERICAL SIMULATION OF MHD STAGNATION POINT FLOW TOWARDS A HEATED AXISYMMETRIC SURFACE
}

\author{
MUHAMMAD ASHRAF ${ }^{凶 1}$ and M. ANWAR KAMAL ${ }^{2}$
}

(Received 13 May, 2009; revised 29 July, 2011)

\begin{abstract}
The problem of stagnation point flow with heat transfer of an electrically conducting fluid impinging normally on a permeable axisymmetric surface in the presence of a uniform transverse magnetic field is analysed. The governing nonlinear differential equations and their associated boundary conditions are reduced to dimensionless form using suitable similarity transformations. Comparison with previously published work shows good agreement. Effects of the injection-suction parameter, magnetic parameter and Prandtl number on the flow and thermal fields are presented. The investigations show that the wall shear stress and heat transfer rate from the surface increase with increased applied magnetic field. An increase in the velocity and thermal boundary layer thicknesses is observed with an increase in the wall injection, while the velocity and thermal boundary layers become thinner when increasing the wall suction and applied magnetic field.
\end{abstract}

2010 Mathematics subject classification: primary 76D05; secondary 76W05, 76D99.

Keywords and phrases: magnetohydrodynamics (MHD); axisymmetric stagnation flow; heat transfer; boundary layer, finite differences.

\section{Introduction}

The hydromagnetic stagnation point flows are fundamental not only in fluid mechanics but also in heat transfer. These flows have attracted many investigators due to their applications in many engineering and natural processes. These include filtration, electronics cooling, blood flow problems, nuclear reactor cooling systems, the paper industry, boundary layers along material handling conveyers, magnetohydrodynamic (MHD) generators, aerodynamic extrusion of plastic sheets, metallurgical and metalworking processes, and the cooling of an infinite metallic plate in a cooling bath.

The classical two-dimensional stagnation point flow on a flat plate introduced by Hiemenz [5] was extended to the axisymmetric case by Homann [7]. Wang [12]

\footnotetext{
${ }^{1}$ Centre for Advanced Studies in Pure and Applied Mathematics, Bahauddin Zakariya University, Multan 60800, Pakistan; e-mail: mashraf_mul@yahoo.com.

${ }^{2}$ Department of Mathematics, Al Kharj University, Al Kharj, Saudi Arabia.

(c) Australian Mathematical Society 2011, Serial-fee code 1446-1811/2011 \$16.00
} 
considered the problem of two dynamic impinging stagnation flows of two fluids of different densities and showed that the flow field depends heavily on the ratio of viscosity and density. The effect of an external magnetic field on Hiemenz flow was investigated by Ariel [1]. The MHD two-dimensional steady stagnation point flow towards a stretching surface was analysed by Mahapatra and Gupta [8], who concluded that the velocity at a point decreases (increases) with an increase in the applied magnetic field when the free stream velocity is less (greater) than the stretching velocity. Chamkha and Issa [3] presented a parametric study of two-dimensional hydromagnetic flow with heat and mass transfer over a permeable flat plate. Sharma and Singh [9] considered the effects of variable thermal conductivity and a heat source or sink on viscous incompressible flow of an electrically conducting fluid in the presence of a uniform transverse magnetic field and variable free stream near a stagnation point on a nonconducting stretching sheet. The laminar boundary layer flow over a horizontal permeable flat plate was studied by Aydın and Kaya [2], who showed that injection causes a decrease in heat transfer while suction enhances the heat transfer coefficient. The stagnation slip flow with heat transfer over a moving plate for two-dimensional and axisymmetric cases was considered by Wang [11], who recently also analysed the problem of axisymmetric stagnation flows and heat transfer towards a shrinking surface [10].

The present study extends the results of the aforementioned authors by considering the effects of an applied magnetic field on the axisymmetric stagnation flow and heat transfer over a permeable axisymmetric surface. A uniform magnetic field is applied to the flow, the magnetic Reynolds number is assumed to be small, and both the viscous and magnetic dissipation are neglected. The governing equations of motion are first reduced to dimensionless form using suitable similarity transformations and then discretized by applying central finite differences.

\section{Problem formulation}

Consider the axisymmetric stagnation point flow of an electrically conducting fluid impinging perpendicularly on a permeable surface. The flow is assumed to be viscous, steady, laminar and incompressible. A uniform transverse magnetic field of strength $\beta_{0}$ is applied to the surface. Then, for the MHD steady axisymmetric stagnation point flow, the governing continuity and momentum equations for boundary layer approximations, including the Lorentz force, can be written in the usual notation as

$$
\begin{gathered}
\frac{\partial u}{\partial x}+\frac{\partial w}{\partial z}=0, \\
u \frac{\partial u}{\partial x}+w \frac{\partial u}{\partial z}=U \frac{d U}{d x}+v{\frac{\partial^{2} u}{\partial z^{2}}+{\frac{\sigma \beta_{0}}{\rho}}^{2}(U-u),}^{2}=
\end{gathered}
$$

where $u$ and $w$ are the velocity components along the $x$ and $z$ axes, respectively, $\rho$ is the density, $v$ the kinematic viscosity and $\sigma$ the electrical conductivity of the fluid, and $U(x)$ is the inviscid potential flow velocity above the boundary layer over the surface. 
The equation for the temperature distribution for the present axisymmetric stagnation point flow problem, neglecting the viscous dissipation, can be written as

$$
\rho c_{p}\left(u \frac{\partial T}{\partial x}+w \frac{\partial T}{\partial z}\right)=k \frac{\partial^{2} T}{\partial z^{2}},
$$

where $c_{p}$ is the specific heat at constant pressure and $k$ is the thermal conductivity of the fluid.

The boundary conditions on the permeable surface for the velocity and temperature fields may be expressed as

$$
\begin{gathered}
u(x, 0)=0, \quad w(x, 0)=-V, \quad u(x, \infty)=U(x)=a x, \\
T(x, 0)=T_{0}, \quad T(x, \infty)=T_{\infty} .
\end{gathered}
$$

Here $V<0$ for injection and $V>0$ for suction, $T_{0}$ and $T_{\infty}$ are temperatures on the surface and at infinity, respectively (with $T_{0}>T_{\infty}$ ), and $a$ is the strength of the stagnation point.

Following Wang [10], we seek a similarity solution of the following form, compatible with the continuity equation (2.1), in order to convert the partial differential equation (2.3) with (2.4) into a system of ordinary differential equations:

$$
u=a x f^{\prime}(\eta), \quad w=-2 \sqrt{v a} f(\eta), \quad \theta(\eta)=\frac{T-T_{\infty}}{T_{0}-T_{\infty}}, \quad \eta=\sqrt{\frac{a}{v}} z .
$$

Here the prime denotes differentiation with respect to $\eta$. In view of (2.5), the continuity equation (2.1) is identically satisfied whereas the momentum and temperature equations (2.2) and (2.3) respectively take the dimensionless form

$$
\begin{gathered}
f^{\prime \prime \prime}+2 f f^{\prime \prime}-f^{2}+M^{2}\left(1-f^{\prime}\right)+1=0, \\
\theta^{\prime \prime}+2 \operatorname{Pr} f \theta^{\prime}=0,
\end{gathered}
$$

where $\operatorname{Pr}$ is the Prandtl number and $M=\sqrt{\sigma \beta_{0}^{2} /(\rho a)}$ is the Hartmann number or magnetic parameter. The boundary conditions (2.4) are reduced (by (2.5)) to

$$
f(0)=A, \quad f^{\prime}(0)=0, \quad f^{\prime}(\infty)=1, \quad \theta(0)=1, \quad \theta(\infty)=0,
$$

where $A= \pm V /(2 \sqrt{v a})$ is the injection-suction parameter, with $A<0$ for injection and $A>0$ for suction. Note that in the absence of a magnetic field $(M=0)$, equations (2.6) and (2.7) reduce to those obtained by Wang [10,12] for the axisymmetric case with different boundary conditions.

The governing equations (2.6) and (2.7), being highly nonlinear, are difficult to solve analytically. We use a finite difference based numerical algorithm to solve them. The third-order equation (2.6) can be converted into one of second order one via the substitution $q=f^{\prime}$, so that (2.6) and the boundary conditions (2.8) take the form

$$
q^{\prime \prime}+2 f q^{\prime}-q^{2}+M^{2}(1-q)+1=0
$$


TABLE 1. Wall shear stress and heat transfer rate for $M=0.4, \operatorname{Pr}=0.7$ and various values of $A$.

$$
\begin{array}{rrrr}
\cline { 2 - 4 } & A & f^{\prime \prime}(0) & -\theta^{\prime}(0) \\
\cline { 2 - 4 }-2.0 & 0.47184 & 0.00113 \\
-1.5 & 0.60387 & 0.01417 \\
-1.0 & 0.81587 & 0.08887 \\
-0.5 & 1.15399 & 0.30664 \\
0 & 1.64528 & 0.69092 \\
0.5 & 2.27726 & 1.19756 \\
1.0 & 3.01620 & 1.77726 \\
1.5 & 3.82944 & 2.39877 \\
2.0 & 4.69281 & 3.04483 \\
\hline
\end{array}
$$

For the numerical solution of the above boundary value problem, (2.7) and (2.9) are discretized at a typical grid point $\eta=\eta_{n}$ of the interval $(0, \infty)$ by employing central difference approximations for the derivatives, and the obtained algebraic system of equations is solved iteratively using the successive over-relaxation (SOR) method [6], subject to the boundary conditions (2.10). Higher-order accuracy for the approximations to the exact solutions can be obtained by using Richardson's extrapolation [4].

\section{Results and discussion}

In this section, we present a comprehensive numerical parametric study of our findings. The results are reported in tabular and graphical forms in order to illustrate the special features of the solutions. We present the effects of the shear stress, injection-suction parameter, applied magnetic field and Prandtl number on the flow and heat transfer characteristics. We note that for $A=0$, the shear stress at the surface is $f^{\prime \prime}(0)=1.311940$, which compares well with the result given by Wang [12] in the absence of a magnetic field (namely $f^{\prime \prime}(0)=1.311938$ ).

The influence of the injection-suction parameter $A$ on the wall shear stress and heat flux at the surface is shown in Table 1 for fixed values of the magnetic parameter $M$ and Prandtl number Pr. Imposition of wall fluid injection $(A<0)$ has the effect of decreasing the wall shear stress and heat loss $\left(-\theta^{\prime}(0)\right)$ from the surface, whereas an opposite effect is noted for wall fluid suction $(A>0)$.

Table 2 gives the values of the wall shear stress and heat flux at the surface for various values of $M$. The effect of increasing the magnetic field is to increase the wall shear stress, due to the increased resistive forces. The heat loss per unit area from the surface also increases with increasing values of $M$. Both wall shear stress and heat loss are larger for suction than for injection.

Table 3 shows that for increasing values of $\mathrm{Pr}$, heat loss from the surface increases in the case of suction and decreases in the case of injection. 


TABLE 2. Wall shear stress and heat transfer rate for $\operatorname{Pr}=0.7$ and various values of
\begin{tabular}{llllrll}
\multicolumn{4}{c}{$A=-1$} \\
\hline$M$ & $f^{\prime \prime}(0)$ & $-\theta^{\prime}(0)$ & $M$ & \multicolumn{4}{c}{$f^{\prime \prime}(0)$} & $-\theta^{\prime}(0)$ \\
\hline 0 & 0.491934 & 0.063328 & 0 & 2.765190 & 1.768272 \\
2 & 1.528229 & 0.130180 & 5 & 6.272546 & 1.869609 \\
4 & 3.300424 & 0.214155 & 10 & 11.124489 & 2.116923 \\
6 & 5.206616 & 0.314523 & 15 & 16.054485 & 2.446776 \\
\hline
\end{tabular}

TABLE 3. Heat transfer rate for $M=1$ and various values of $\operatorname{Pr}$.

\begin{tabular}{ccc}
\hline Pr & $-\theta^{\prime}(0)$ with $A=-1$ & $-\theta^{\prime}(0)$ with $A=1$ \\
\hline 0.1 & 0.211952 & 0.515508 \\
0.2 & 0.190000 & 0.731000 \\
0.3 & 0.167000 & 0.949000 \\
0.4 & 0.144000 & 1.162227 \\
0.5 & 0.123855 & 1.370879 \\
0.6 & 0.105000 & 1.575549 \\
0.7 & 0.089000 & 1.777295 \\
\hline
\end{tabular}

We also give graphical representations of the velocity and temperature profiles to investigate the influences of the parameters $A, M$ and Pr on the flow and thermal fields. The velocity profile $f^{\prime}(\eta)$ for various values of $A$ is predicted in Figure 1(a). The velocity profiles fall when increasing the injection $(A<0)$, and the opposite behaviour is observed for suction $(A>0)$. The velocity boundary layer thickness increases with increased injection, and decreases when increasing the suction at the surface. The boundary layer thickness for injection is larger than that for suction. The effect of $A$ on the thermal field is shown in Figure 1(b). Increasing the injection serves to increase the thermal boundary layer thickness. On the other hand, the thermal boundary layer thickness decreases with increasing positive values of $A$.

The influence of imposing an external magnetic field on the velocity and thermal fields is shown in Figures 2 and 3. The velocity profiles increase when increasing $M$, for any value of $A$. Due to the damping effects, the velocity boundary layer thickness decreases with increasing values of the applied magnetic field for both the injection and suction cases. A decrease in the temperature is observed with an increase in $M$. The thermal boundary layer thickness decreases with increasing $M$, for any $A$.

Finally, the effect of the Prandtl number on the temperature is shown in Figure 4 for given values of $A$ and $M$. Increasing the value of Pr increases the thermal boundary layer thickness in the case of wall injection $(A<0)$ in a region near the surface, and the opposite effect is observed in the case of wall suction.

We have observed that the effect of the governing parameters on the velocity and temperature fields is more significant in the case of injection as compared to suction. 

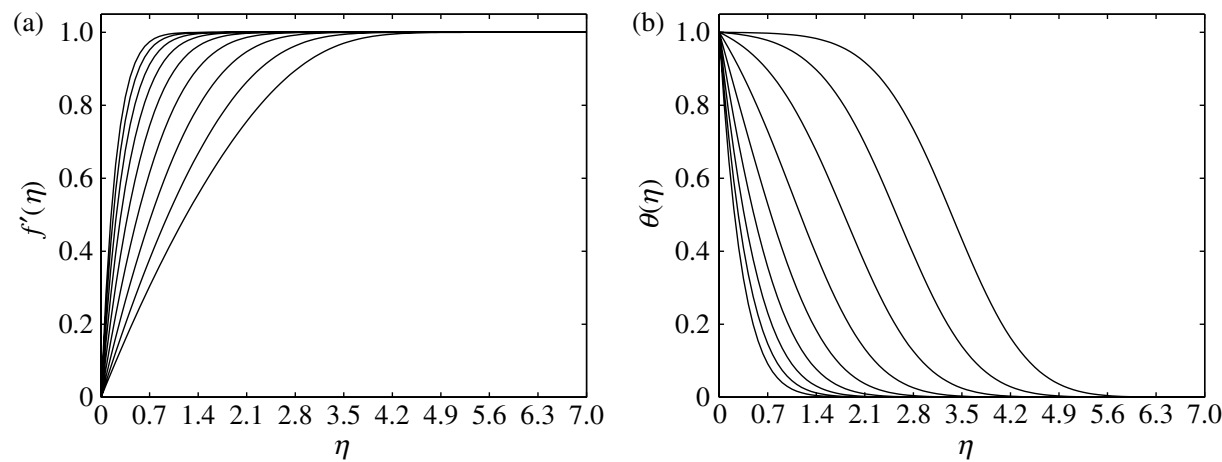

FIgURE 1. (a) Velocity profiles $f^{\prime}(\eta)$ and (b) temperature profiles $\theta(\eta)$, for $M=1.0$ and $\operatorname{Pr}=0.7$. In each case, the right-most curve corresponds to $A=-2$ and then $A$ increases from right to left through the values $-1.5,-1,-0.5,0,0.5,1,1.5,2$.
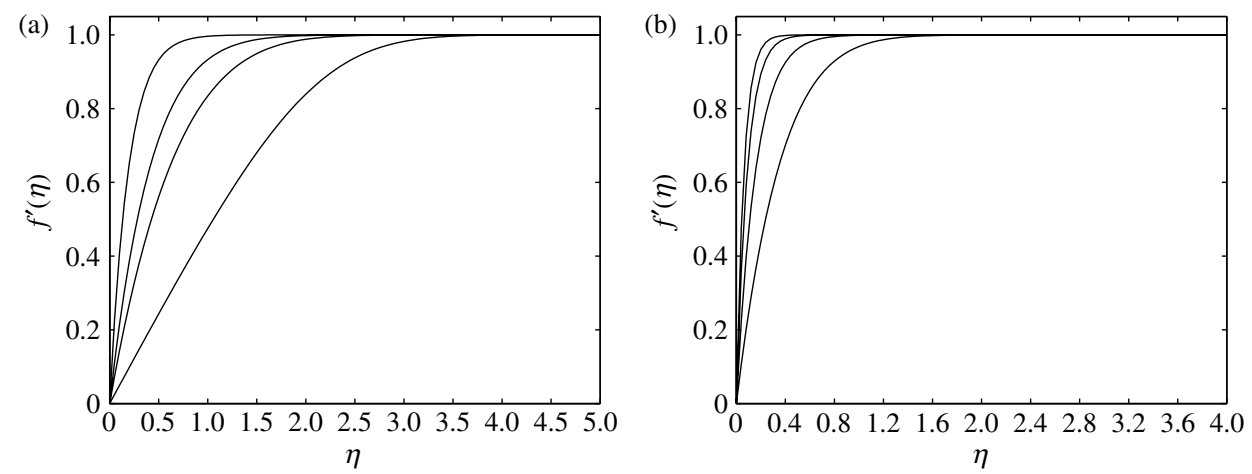

FIGURE 2. Velocity profiles $f^{\prime}(\eta)$ for (a) $A=-1$ (injection) and (b) $A=1$ (suction), with $\operatorname{Pr}=0.7$. In each case, the right-most curve corresponds to $M=0$ and then $M$ increases from right to left through the values (a) 2, 4, 6 and (b) 5, 10,15.
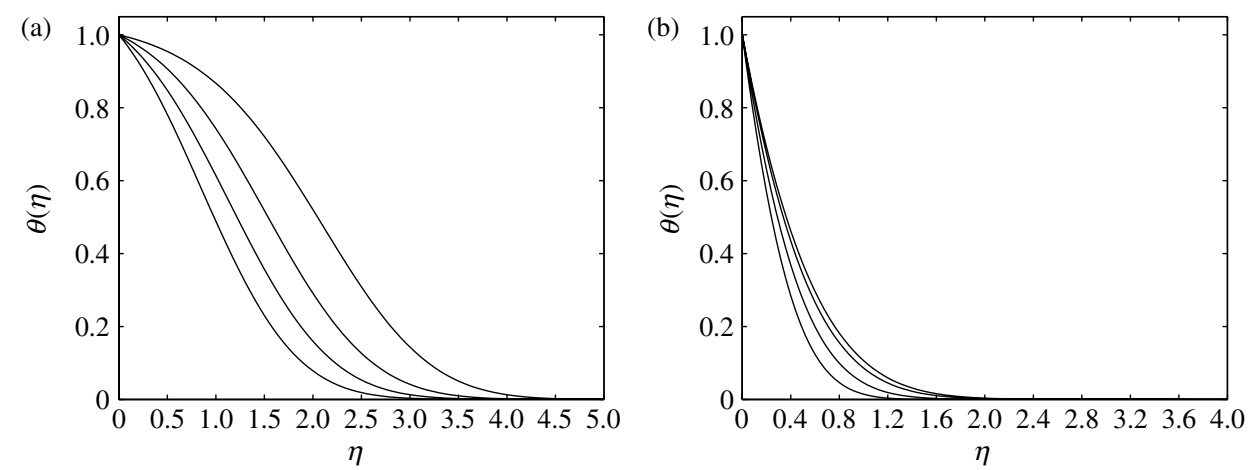

FIgURE 3. Temperature profiles $\theta(\eta$ ) for (a) $A=-1$ (injection) and (b) $A=1$ (suction), with $\operatorname{Pr}=0.7$. In each case, the right-most curve corresponds to $M=0$ and then $M$ increases from right to left through the values (a) 2, 4, 6 and (b) 5, 10, 15. 

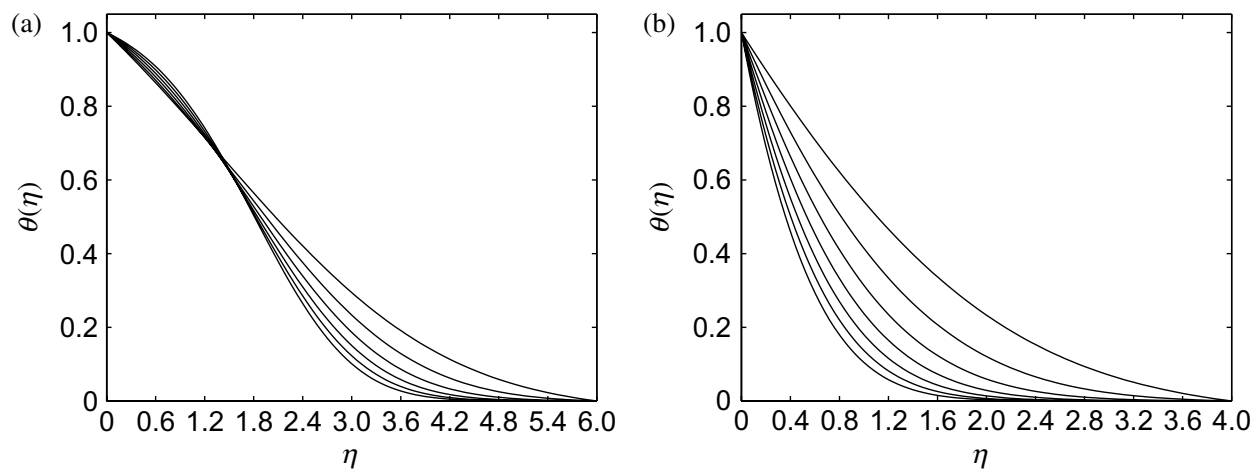

Figure 4. Temperature profiles $\theta(\eta$ ) for (a) $A=-1$ (injection) and (b) $A=1$ (suction), with $M=1.0$. In each case, the right-most curve corresponds to $\operatorname{Pr}=0.1$ and then $\operatorname{Pr}$ increases from right to left through the values $0.2,0.3,0.4,0.5,0.6,0.7$.

\section{Conclusions}

This work considered the effects of the injection-suction parameter, the magnetic parameter, and the Prandtl number on the steady, laminar, axisymmetric stagnation flow of an electrically conducting fluid with heat transfer over a permeable surface. The self-similar equations with boundary conditions governing the fluid flow and temperature were solved numerically using an algorithm based on finite differences. The investigations show that increasing the wall fluid injection has the tendency to decrease the wall shear stress and heat loss from the surface, whereas an opposite effect is noted for wall fluid suction. The wall shear stress and heat loss from the surface increase when increasing the magnetic parameter $M$. Increasing the value of Pr has the influence of increasing the heat loss but has no effect on the wall shear stress. The applied magnetic field enhances the velocity and thermal boundary layer thicknesses. Increasing the value of Pr increases the thermal boundary layer thicknesses in the case of injection, while an opposite effect is noted in case of suction.

\section{Acknowledgements}

The authors wish to express their sincere thanks to Dr. Andrew Bassom and the reviewers for their valuable suggestions to improve the quality of the paper.

\section{References}

[1] P. D. Ariel, "Hiemenz flow in hydromagnetics", Acta Mech. 103 (1994) 31-43; doi:10.1007/BF01180216.

[2] O. Aydın and A. Kaya, "Laminar boundary layer flow over a horizontal permeable flat plate", Appl. Math. Comput. 161 (2005) 229-240; doi:10.1016/j.amc.2003.12.021.

[3] A. J. Chamkha and C. Issa, "Effects of heat generation/absorption and thermophoresis on hydromagnetic flow with heat and mass transfer over a flat surface", Int. J. Numer. Methods Heat Fluid Flow 10 (2000) 432-449; doi:10.1108/09615530010327404. 
[4] P. Deuflhard, "Order and stepsize control in extrapolation methods", Numer. Math. 41 (1983) 399-422; doi:10.1007/BF01418332.

[5] K. Hiemenz, "Die Grenzschicht an einem in den gleichförmingen Flüssigkeitsstrom eingetauchten graden Kreiszylinder", Dinglers Polytech. J. 326 (1911) 321-324.

[6] F. B. Hildebrand, Introduction to numerical analysis (Tata McGraw-Hill, New Delhi, 1979).

[7] F. Homann, "Der Einfluss grosser Zähigkeit bei der Strömung um den zylinder und um die Kugel", Z. Angew. Math. Mech. 16 (1936) 153-164.

[8] T. R. Mahapatra and A. S. Gupta, "Magnetohydrodynamic stagnation-point flow towards a stretching sheet", Acta Mech. 152 (2001) 191-196; doi:10.1007/BF01176953.

[9] P. R. Sharma and G. Singh, "Effects of variable thermal conductivity and heat source/sink on MHD flow near a stagnation point on a linearly stretching sheet", J. Appl. Fluid Mech. 2 (2009) 13-21.

[10] C. Y. Wang, "Stagnation flow towards a shrinking sheet", Int. J. Non-Linear Mech. 43 (2008) 377-382; doi:10.1016/j.ijnonlinmec.2007.12.021.

[11] C. Y. Wang, "Stagnation slip flow and heat transfer on a moving plate", Chem. Eng. Sci. 61 (2006) 7668-7672; doi:10.1016/j.ces.2006.09.003.

[12] C. Y. Wang, "Impinging stagnation flows", Phys. Fluids 30 (1987) 915-917; doi:10.1063/1.866345. 